Nloman 2015, 33(2), 77-83

Revista de Psicologia, Ciències de l'Educació i de l'Esport

ISSN: 1138-3194

Copyright (C) 2015

www.revistaaloma.net

\title{
Nuevas tecnologías - ¿Nuevas patologías? El smartphone y el fear of missing out
}

\author{
Frederic Gil, ${ }^{1}$ Georgina del Valle, ${ }^{1}$ Ursula Oberst ${ }^{1}$ y Andrés Chamarro ${ }^{2}$ \\ ${ }^{1}$ Universitat Ramon Llull, ${ }^{2}$ Universitat Autònoma de Barcelona
}

Recibido: 3-9-2015

Aceptado: 5-10-2015

\section{Nuevas tecnologías - ¿Nuevas patologías? El smartphone y el fear of missing out}

Resumen. En el contexto de las investigaciones sobre el uso intensivo y/o maladaptativo del teléfono móvil y de las redes sociales en línea se han ido describiendo nuevos fenómenos que pueden ser asociados a un malestar psicológico importante. El fear of missing out o FoMO (miedo a perderse algo) es un nuevo constructo psicológico que se ha descrito como la sensación de malestar al saber que otras personas están realizando actividades agradables y que uno no es parte de ello. El presente estudio investiga la relación entre el grado de FoMO experimentado, los indicadores de uso problemático del teléfono móvil y los indicadores de malestar psicológico. Mediante un análisis de regresión lineal jerárquico se encontró que tanto la conducta adictiva en torno al teléfono móvil como el FoMO son importantes predictores de síntomas psicopatológicos, especialmente, de ansiedad. El fear of missing out se perfila como un correlato importante del uso desadaptativo del smartphone y de las redes sociales en línea.

Palabras clave: redes sociales en línea; fear of missing out; FoMO, smartphone; teléfono inteligente; adicción; uso intensivo

New technologies - new disorders? The smartphone and the fear of missing out

Summary. In the context of research on heavy or maladaptive use of mobile phones and online social networks new phenomena have been described that can be associated with significant psychological distress. The fear of missing out or FoMO is a new psychological construct that has been described as the feeling of distress that people have when learning that other people are doing enjoyable activities and one is not part of it. This study investigates the relationship between the degree of experienced FoMO, indicators of problematic mobile phone use and indicators of psychological distress. Using a hierarchical linear regression analysis it was found that both addictive behavior in relation to the mobile phone and FoMO are important predictors of psychopathological symptoms, especially anxiety. The fear of missing out is emerging as an important correlate of maladaptive use of smartphones and online social networks.

Keywords: online social networks; fear of missing out; FoMO; smartphone; addiction; heavy use

Correspondencia

Ursula Oberst

FPCEE Blanquerna

Universitat Ramon Llull

c/Císter, 34, 08022 Barcelona

Email: ursulao@blanquerna.url.edu 


\section{Introducción}

Las tecnologías de la información y la comunicación (TIC) son el conjunto de recursos, procedimientos y técnicas usadas en el procesamiento, el almacenamiento y la transmisión de información. Entre ellos encontramos los smartphones, que ofrecen múltiples recursos y ventajas a sus usuarios e influyen en la forma en la que estos se relacionan entre sí y perciben el mundo que les rodea. Las TIC actuales permiten que una persona pueda permanecer conectada constantemente sin que importe el lugar en el que se encuentre, en otras palabras, le permite estar constantemente conectada (Hardey, 2011). A pesar de las múltiples ventajas que tienen estas tecnologías, su abuso puede tener un fuerte impacto en la persona, con consecuencias en los campos psicofisiológico, afectivo y socioeconómico (Carbonell et al., 2012; Billieux, Maurage, López-Fernández, Kuss \& Griffiths, 2015; Kuss, Griffiths \& Binder, 2013; Renau, Gil, Oberst \& Carbonell, 2015). Es por ello por lo que, durante los últimos 15 años, han sido numerosos los estudios que se han centrado en estudiar más en profundidad este fenómeno, para poder determinar las causas, la incidencia y la frecuencia de las «adicciones tecnológicas» (Griffiths, 1995). En una primera aproximación a este tema, Young (1998) expuso las principales señales de alarma que denotan una dependencia a Internet y que pueden reflejar el paso de afición a adicción. Entre ellas encontramos: a) privarse del sueño para estar conectado a la Red, a la que se dedica un tiempo de conexión anormalmente alto; b) descuidar otras actividades importantes, como el contacto con la familia, las relaciones sociales, el estudio o el cuidado de la salud; c) recibir quejas en relación con el uso excesivo de la Red por parte de alguien cercano, como los padres o hermanos; d) pensar en la Red constantemente, incluso cuando no se está conectado a ella, y sentirse irritado excesivamente cuando la conexión falla o resulta muy lenta; e) intentar limitar el tiempo de conexión, pero sin conseguirlo, y perder la noción del tiempo; f) mentir sobre el tiempo real que se está conectado o jugando a un videojuego; g) aislarse socialmente, mostrarse irritable y bajar el rendimiento en los estudios; y h) sentir una euforia y activación anómalas cuando se está delante del ordenador.

Aun así, entre los investigadores existe controversia sobre si la adicción a las TIC, sea a Internet, los videojuegos en línea, el teléfono móvil o las redes sociales en línea, realmente existe o únicamente se puede hablar de abuso, uso inadecuado o uso intensivo (Chóliz, 2010; Billieux et al., 2015; Pontes, Szabo \& Griffiths, 2015). Si bien es cierto que el abuso podría desencadenar un uso patológico, el debate está en si este uso patológico puede derivar en una adicción y, de ser así, debería incluirse dentro de la categoría de trastornos no relacionados con sustancias o adicciones conductuales, o bien, mantenerse al margen (Young, 2015). En la quinta edición del Manual diagnóstico y estadístico de los trastornos mentales (APA, 2013) la clasificación diagnóstica propuesta para esta categoría incluye únicamente el juego patológico, aunque contempla la adicción a los videojuegos en línea como categoría que requiere mayor consideración. La adicción a Internet o al teléfono móvil no queda contemplada. A pesar de ello, son muchos los autores que exponen en sus estudios que la adicción a las nuevas tecnologías existe, pues se ha constatado la aparición de síntomas similares que a los dados en la adicción a sustancias.

Recientemente, algunas de las investigaciones sobre las consecuencias negativas de las TIC se han centrado en las redes sociales online (RSO), como Facebook, Instagram, Twitter o WhatsApp. Se ha mostrado que su uso inadecuado o abusivo puede producir un malestar psicológico y una sintomatología que son comparables a una adicción, sobre todo porque, en la actualidad, los smartphones permiten a los usuarios mantenerse conectados sin que importe el tiempo ni el lugar (Renau, Gil, Oberst \& Carbonell, 2015). Por encima de todo, las investigaciones han evidenciado que son los más jóvenes los que son más vulnerables a la hora de desarrollar un uso problemático (Kuss et al., 2013; Przybylski, Murayama, DeHaan \& Gladwell, 2013; Renau et al., 2015). Durante la adolescencia y adultez temprana es cuando se empieza desarrollar la necesidad de pertenencia al grupo y las redes sociales ofrecen una manera de amplificar la sensación de conexión con las personas. Aunque algunos autores exponen que no podemos hablar de adicción al teléfono móvil y prefieren hablar de uso abusivo, intensivo, problemático o desadaptativo (Sánchez-Carbonell, Beranuy, Castellana, Chamarro \& Oberst, 2008), se ha mostrado que asociados a estas conductas encontramos síntomas como la irritación, el nerviosismo, la inquietud, la agresividad o la excesiva preocupación cuando su uso no es posible, mientras que la utilización produce sensación de relajación. Así, algunos autores (Pedrero, Rodríguez \& Ruiz, 2012) consideran que las consultas frecuentes para comprobar si se han recibido nuevos mensajes o llamadas podrían indicar el grado de dependencia del móvil. A esto cabe añadir que las mujeres perciben el uso del teléfono como más problemático y que los hombres (Beranuy, Oberst, Carbonell \& Chamarro, 2009; Chóliz, Villanueva \& Chóliz, 2009; Jenaro, Gómez-Vela, González-Gil \& Caballo, 2007; Labrador \& Villadangos, 2010). Según Chóliz, Villanueva y Chóliz (2009), las chicas utilizan más el móvil para enfrentarse a estados de ánimo displacenteros, y un factor que emerge como indicador de uso problemático son las consultas frecuentes del móvil, lo que podría indicar la importancia del acceso a las redes sociales (Labrador \& Villadangos, 2010).

\section{El fear of missing out (el miedo a quedarse fuera)}

En este contexto, algunos investigadores han propuesto nuevos desórdenes. Por ejemplo, se ha propuesto la existencia de nomofobia (del inglés, no-mobile-phonephobia, King et al., 2013; Bragazzi \& Del Puente, 2014), que designa el sentimiento de miedo irracional que 
experimentan muchas personas al salir a la calle sin su teléfono, quedarse sin batería o estar en un lugar con poca o ninguna cobertura de la red. Según los estudios, alrededor del 58\% de los hombres y el $48 \%$ de las mujeres sufrían este trastorno, y un 9\% adicional se sentía estresado cuando sus móviles estaban apagados. El 55\% afirmó que por el hecho de estar «aislado» de las posibles llamadas o mensajes de familiares y amigos experimentaban ansiedad, mientras que un escaso $10 \%$ afirmó que la causa era su trabajo, ya que exigía una conexión permanente.

Otro nuevo fenómeno descrito es el ringxiety, también conocido como phantomringing o ruido fantasma (Avvannavar, Kumar, Shrihari \& Babu Are, 2008). Se experimenta cuando la persona oye sonar su teléfono o nota su vibración cuando este en realidad no suena. Este fenómeno se relaciona con dolores de cabeza, estrés, alteraciones del sueño y depresión (Oftedal, Wilen, Sandstromt \& Mild, 2000; Thomée, Harenstam \& Hagberg, 2011). El estudio de Thomée et al. (2011) reveló el ringxiety como factor de riesgo en el desarrollo de problemas relacionados con la salud mental.

En la misma línea, el concepto de FoMO (fear of missing out) ha ganado mucha popularidad durante los últimos años. Este término fue acuñado por Dan Herman, pero no ha ganado fuerza hasta la publicación de Przybylski, Murayama, DeHaan y Gladwell (2013), en la cual lo describen como una sensación de malestar que puede llegar a sentirse al saber que otras personas están realizando actividades agradables y uno no forma parte de ello. En este estudio se encontró una correlación entre el FoMO y el malestar psicológico general. Przybylski et al. eran los primeros en facilitar una comprensión operacionalizada y empíricamente validada del fenómeno FoMO, al crear un cuestionario de auto-informe y facilitar la evidencia empírica de su validez de constructo y de sus correlatos psicológicos.

Los usuarios afectados por el FoMO temen quedarse al margen y sentirse excluidos por no haber estado pendientes de lo que estaba pasando en las redes sociales en línea. El FoMO no puede considerarse una forma de adicción a las redes sociales, pero puede ser un catalizador del uso desadaptativo, y de cómo las redes sociales en línea pueden convertirse en fuentes de estrés y ansiedad que pueden perjudicar la salud de la persona.

Según indican Przybylski et al. (2013), el FoMO está relacionado con la regulación afectiva. El FoMO mostró estar relacionado con un mayor uso de las redes sociales, menor satisfacción de las necesidades psicológicas básicas (competencia, autonomía, estar conectado con otros) y menor satisfacción en la vida. El fenómeno FoMO se comprendería como un mecanismo autorregulador surgido como consecuencia de la insatisfacción crónica o puntual de las necesidades básicas (Alt, 2015).

De acuerdo con lo expuesto anteriormente, el objetivo del presente estudio es investigar si existe una relación entre el grado de FoMO experimentado, los indicadores de uso problemático del teléfono móvil y los indicadores de malestar psicológico. Se espera en- contrar que tanto el uso intensivo y desadaptativo de las redes sociales mediante el smartphone, como el FoMO se perfilen como predictores del malestar psicológico.

\section{Método}

\section{Participantes}

El estudio se llevó a cabo con una muestra autoseleccionada de 289 españoles (95 hombres, 194 mujeres), con una edad media de 22.95 años ( $\mathrm{SD}=10.19$ ) que contestaron un cuestionario en línea. La media de redes sociales utilizadas era de 3.4 redes $(\mathrm{SD}=1.4)$, la más utilizada de las cuales fue Facebook (96.2\% de la muestra).

\section{Instrumentos}

Uso problemático del teléfono móvil. Se ha utilizado el Cuestionario de Experiencias Relacionadas con el Móvil (CERM, Beranuy, Chamarro, Graner \& Carbonell, 2009) para la evaluación del grado de adicción al móvil. Está formado por 10 ítems con 4 posibles respuestas tipo Likert que van de 1 (casi nunca) a 4 (casi siempre). El presente estudio mostró una consistencia interna de $\alpha$ $=.86$.

Fear of missing out. Para evaluar el FoMO se ha usado la adaptación española del Cuestionario de fear of missing out (Gil, Chamarro \& Oberst, 2015). La escala evalúa los miedos y las preocupaciones que el individuo puede experimentar en relación con estar fuera de contacto con las experiencias de su entorno social extendido. Consta de 10 ítems con 5 posibles respuestas tipo Likert que van de 1 (nada) a 5 (mucho) y evalúan los sentimientos y las emociones que experimenta la persona en sus interacciones con los demás y su percepción de estas. El presente estudio mostró una consistencia interna de $\alpha=.85$.

Uso de las redes sociales en línea: intensidad, frecuencia $y$ tipo de acceso. Se han utilizado tres cuestionarios: 1) El Social media engagement questionnaire (SMEQ) desarrollado por Przybylski et al. (2013). Consta de 5 ítems con una escala de Likert de 7 puntos que miden el uso de las redes sociales en línea durante el día a día. Las puntuaciones se suman para obtener un índice de implicación (engagement) con las redes sociales. 2) Para la intensidad de uso, se aplicó la escala Social network intensity (SNI) de Salehan \& Negahban (2013), compuesta por 5 ítems en una escala de Likert de 7 puntos que van de «nunca» a «siempre». 3) Del mismo estudio se tomó la escala Social network access via mobile phone applications (SNMA) para medir el grado en el que los participantes usaron el smartphone para acceder a las redes. Cuenta con 5 ítems en una escala de Likert de 7 puntos.

Malestar emocional. Se ha usado la escala de ansiedad y depresión hospitalaria (HADS), traducida y adaptada al español por Quintana et al. (2003). Consta de dos subescalas de 7 ítems cada una: una para medir la an- 
siedad y otra para la depresión, con 4 alternativas de respuesta. Las puntuaciones para la escala entera (malestar emocional) varían entre 0 y 42 ; puntuaciones más altas indican mayor malestar. El mejor ajuste de sensibilidad y especificidad para la escala total eran puntuaciones superiores a 13 (Singer et al., 2009). El presente estudio mostró una consistencia interna de $\alpha$ $=.738$ (para ansiedad), $\alpha=.586$ (para depresión), y $\alpha$ $=.763$ (escala HADS total).

\section{Procedimiento}

La recogida de datos se realizó a través de un cuestionarios en línea, (www.surveygizmo.com), configurado para que la obtención de respuestas se realizara de forma totalmente anónima. La captación de la muestra se realizó a través de una página de Facebook (www.facebook.com/spanishfomo) que daba acceso al enlace del cuestionario. Los participantes eran animados a colaborar mediante un enlace (www.midetufomo. com) y contestando el cuestionario. Tras responder el cuestionario, los participantes recibían feedback sobre su grado de FoMO, basado en parámetros estadísticos (medias y cuartiles).

\section{Análisis de datos}

Se efectuaron pruebas $t$ para diferencias de género y correlaciones entre las variables. También se efectuó un análisis de regresión múltiple de las variables FoMo, CERM, SNI, SNMA y SMEQ para predecir el malestar emocional. Los análisis se realizaron con el programa SPSS versión 17.

\section{Resultados}

\section{Estadísticos descriptivos}

Los estadísticos descriptivos pueden ser consultados en la tabla 1. Las puntuaciones del uso abusivo del móvil en nuestra muestra son menores que en el estudio original con adolescentes (Beranuy et al. 2009). Las puntuaciones del SMEQ y del FoMO también son inferiores a la media esperada de la escala. En cambio, las puntuaciones de intensidad de uso de las redes sociales (SIN) son superiores a la media, y la de acceso a las redes a través del teléfono móvil (SNMA) muy superio-

Tabla 1. Estadísticos descriptivos de las variables del estudio

\begin{tabular}{lcccl}
\hline \multirow{2}{*}{ Variables } & \multicolumn{2}{c}{ Rango } & Media & SD \\
\cline { 2 - 3 } & Mín. & Máx. & & \\
\hline FOMO & 10 & 49 & 20.43 & 6.68 \\
CERM & 10 & 40 & 16.88 & 5.2 \\
SMEQ & 0 & 35 & 15.43 & 9.21 \\
SNMA & 5 & 35 & 28.07 & 8.89 \\
SNI & 5 & 35 & 22.77 & 7.02 \\
HADS-A & 0 & 19 & 6.87 & 4.23 \\
HADS-D & 0 & 14 & 3.83 & 3.10 \\
HADS & 0 & 32 & 10.70 & 6.59 \\
\hline
\end{tabular}

res. En cuanto al HADS, considerando los puntos de corte existentes, 4 participantes (1.4\%) presentan niveles clínicos de ansiedad, 69 (23.9\%) serían casos posibles, y 178 (74.7\%) con niveles no clínicos. Para la depresión, 38 participantes (13.8\%) presentan niveles clínicos, 134 (46.4\%) eran casos posibles, y 107 (40.5\%) fueron clasificados con niveles no clínicos. Para el malestar emocional, $94(43,8 \%)$ pueden ser considerados sujetos afectados.

Existen diferencias de género para SNI, SNMA y CERM. Las mujeres presentan niveles más altos de intensidad de uso $(t=-3.64, p=.000, d f=287)$, de acceso a través del smartphone $(t=-4.68, p=.000, d f=$ 287) y de adicción al móvil $(t=-2.70, p=.007, d f=$ 287).

\section{Correlaciones}

Los valores del coeficiente de correlación (tabla 2) indican que la edad y el sexo correlacionan de forma baja con las medidas de uso abusivo del móvil, la intensidad de uso de las redes sociales, la ansiedad y la depresión. En cambio, las correlaciones entre las medidas de uso abusivo del móvil y el tipo uso de redes sociales, la intensidad de uso las redes sociales y la ansiedad son moderadas. Las correlaciones con la depresión son sistemáticamente menores que con la ansiedad.

\section{Predicción de malestar emocional}

Se adaptó un modelo de regresión lineal múltiple para predecir el malestar emocional basado en las variables CERM, FoMO, SNI, SNMA y SMEQ. Se encontró una ecuación de regresión significativa con $\mathrm{R} 2=.210$, con lo que el modelo resultante explica el $21 \%$ de la varianza de la puntuación del HADS. El modelo (tabla 3) indica que el CERM y el FoMO predicen el malestar psicológico en alto grado, y el SNI poco y negativamente.

\section{Discusión}

En este estudio se quiso investigar la relación entre el fear of missing out y los indicadores de uso problemático del teléfono móvil con el malestar psicológico. Los resultados demuestran que tanto la conducta adictiva como el FoMO son predictores importantes de malestar emocional.

La necesidad de pertenecer y el deseo de estar conectado socialmente, saber qué hacen los demás e intentar formar parte de estas experiencias son probablemente necesidades básicas presentes desde la infancia (Adler, 1912/1993; Baumeister \& Leary, 1995) y por ello, no restringido al uso de las redes sociales en línea. Pero estas aplicaciones tecnológicas pueden contribuir a un aumento de esta necesidad de pertenecer, incluso cuando en realidad se tiene poco contacto con los otros usuarios (Krämer, Hoffmann \& Eimler, 2015), porque han incrementado exponencialmente las posibilidades de conectarse, compartir y tener experiencias gratifi- 
Tabla 2. Correlaciones entre las variables del estudio

\begin{tabular}{|c|c|c|c|c|c|c|c|c|}
\hline & Sexo & Edad & CERM & SMEQ & FoMO & SNI & HADS-A & HADS-D \\
\hline Sexo & 1 & $-.177^{* *}$ & $.157^{* *}$ & $.226^{\star *}$ & .044 & $.210^{\star *}$ & .087 & -.076 \\
\hline Edad & & 1 & $-.338^{* *}$ & $-.166^{\star *}$ & $-.295^{\star *}$ & $-.271^{\star \star}$ & $-.192^{\star \star}$ & -.021 \\
\hline CERM & & & 1 & $.409^{\star *}$ & $.516^{\star *}$ & $.514^{\star *}$ & $.410^{\star \star}$ & $.253^{\star *}$ \\
\hline SMEQ & & & & 1 & $.222^{\star *}$ & $.384^{\star \star}$ & $.205^{\star \star}$ & $.131^{\star *}$ \\
\hline FoMO & & & & & 1 & $.489^{* *}$ & $.406^{* *}$ & $.226^{\star *}$ \\
\hline SNI & & & & & & 1 & $.201^{\star *}$ & .020 \\
\hline HADS-A & & & & & & & 1 & $.603^{\star *}$ \\
\hline HADS-D & & & & & & & & 1 \\
\hline
\end{tabular}

Tabla 3. Resumen del análisis de regresión múltiple para malestar emocional $(n=289)$

\begin{tabular}{|c|c|c|c|c|c|}
\hline Variable & B & SE(B) & beta & $t$ & $\mathrm{p}$ \\
\hline (constant) & 1,878 & 1.46 & & 1.28 & .200 \\
\hline CERM & .382 & 0.087 & .302 & 4.38 & .000 \\
\hline FoMO & .284 & .064 & .288 & 4.44 & .000 \\
\hline SNI & -.158 & .069 & -.169 & -2.31 & .022 \\
\hline SNMA & -.026 & .049 & -.035 & -.53 & .599 \\
\hline SMEQ & .059 & .043 & .083 & 1.37 & .170 \\
\hline
\end{tabular}

cantes debido a la accesibilidad permanente a través de dispositivos móviles, independientemente del lugar y de la hora. Esto puede, por un lado crear una necesidad permanente de búsqueda de información y, por tanto, incrementar la frecuencia y el engagement con estas tecnologías; los individuos con un mayor grado de FoMO pueden sentirse más obligados de revisar constantemente sus redes sociales para mantenerse actualizados con los planes y las actividades de sus amigos. Pero, por otro lado, el uso intensivo de las redes sociales puede, a su vez, disparar el miedo a perderse algo, porque los usuarios perciben las posibilidades aparentemente ilimitadas que ofrecen las aplicaciones. Se crea una especie de retroalimentación entre uso intensivo o maladaptativo y FoMO, y por eso, la llamada «adicción» a las redes sociales en línea también puede verse agravada por la presencia de FoMO. Futuros estudios deben refinar en los mecanismos subyacentes que existen en la relación entre ambos fenómenos.

\section{Referencias}

Adler, A. (1912/1993). El carácter neurótico. Barcelona: Paidós.

Alt, D. (2015). College students' academic motivation, media engagement and fear of missing out. Computers in Human Behavior, 49, 111-119.

American Psychiatric Association (2014). DSM-V. Manual diagnóstico y estadístico de los trastornos mentales. Editorial Médica Panamericana.

Avvannavar S., Kumar B.S., Shrihari S. \& Babu Are R. (2008). Mobile phones: an anthropological review of its Evolutionary impact. The Journal of International Social Research, Volume 1/5.
Beranuy, M., Chamarro, A., Graner, C. \& Carbonell, X. (2009). Validación de dos escalas breves para evaluar la adicción a Internet y el abuso de móvil. Psicothema, 21, 480-485.

Baumeister, R. F. \& Leary, M. R. (1995). The need to belong: Desire for interpersonal attachments as a fundamental human motive. Psychological Bulletin, 117, 497-529.

Beranuy, M., Oberst, U., Carbonell, X. \& Chamarro, A. (2009). Problematic Internet and mobile phone use and clinical symptoms in college students: The role of emotional intelligence. Computers in Human Behavior 25, 1182-1187.

Billieux, J., Maurage, P., López-Fernández, O., Kuss, D. J., \& Griffiths, M. D. (2015). Can disordered mobile phone use be considered a behavioral addiction? An update on current evidence and a comprehensive model for future research. Current Addiction Reports, 2(2), 156-162.

Bragazzi, N. L., \& Del Puente, G. (2014). A proposal for including nomophobia in the new DSM-V. Psychology research and behavior management, 7, 155-160.

Carbonell, X., Chamarro, A., Beranuy, M., Griffiths, M., Oberst, U., Cladellas, R y Talarn, A. (2012). Problematic Internet and cell phone use in Spanish teenagers and young students. Anales de Psicologia, 28, 789-796.

Chóliz, M. (2010). Mobile phone addiction: a point of issue. Addiction, 105, 373-374.

Chóliz, M., Villanueva, V. \& Chóliz, M.C. (2009). Ellos, ellas y su móvil: Uso, abuso (y dependencia?) del teléfono móvil en la adolescencia. Revista Española de Drogodependencias, 34(1), 74-88.

Gil, F., Chamarro A. \& Oberst, U. (2015). Addiction to 
online social networks: A question of «Fear of Missing Out»? Journal of Behavioral Addictions 4(Suppl. 1), pp. 1-66 (2015). DOI: 10.1556/JBA.4.2015.Suppl.1

Griffiths, M.D. (1995). Technological addictions. Clinical Psychology Forum, 76, 14-19.

Hardey, M. (2011). ICTs and generations: constantly connected social lives. En Broadband Society and Generational Changes (pp. 217). Berna, Suiza: Peter Lang. Jenaro, C., Gomez-Vela, M., Gonzalez-Gil, F. \& Caballo, C. (2007). Problematic Internet and cell phone use: Psychological, behavioural, and health correlates. Addiction Research and Theory, 15(3), 309-320.

King, A. L. S., Valença, A. M., Silva, A. C. O., Baczynski, T., Carvalho, M. R., \& Nardi, A. E. (2013). Nomophobia: Dependency on virtual environments or social phobia?. Computers in Human Behavior, 29(1), 140144.

Krämer, N., Hoffmann, L. \& Eimler, S. (2015). Not Breaking Bonds on Facebook-Mixed-Methods Research on the Influence of Individuals' Need to Belong on «Unfriending'Behavior on Facebook. International Journal of Developmental Science, 9(2), 61-74.

Kuss, D. J., Griffiths, M. D., \& Binder, J. F. (2013). Internet addiction in students: Prevalence and risk factors. Computers in Human Behavior, 29, 959-966. doi:10.1016/j.chb.2012.12.024

Labrador, F. J. \& Villadangos, S. M., (2010). Menores y nuevas tecnologías: conductas indicadoras de posible problema de adicción. Psicothema, 22(2), 180-188.

Oftedal, G., Wilen, J., Sandstromt, M. \& Mild K.H. (2000). Symptoms experienced in connection with mobile phone use. Occupational Medicine, 50(4), 237 45.

Pedrero, J.E., Rodríguez, M.T. \& Ruiz, J.M. (2012). Adicción o abuso del teléfono móvil. Revisión de la literatura. Adicciones, 24, 139-152.

Pontes, H. M., Szabo, A., \& Griffiths, M. D. (2015). The impact of Internet-based specific activities on the perceptions of Internet addiction, quality of life, and excessive usage: A cross-sectional study. Addictive Behaviors Reports, 1, 19-25.

Przybylski, A.K., Murayama, K., DeHaan C.R. \& Gladwell, V. (2013). Computers in Human Behaviour: Motivational, emotional, and behavioral correlates of fear of missing out. Elsevier: Computers in Human Behaviour, 29, 1841-1848.

Quintana, J.M., Padierna, A., Esteban, C, Arostegui, I., Bilbao, A. \& Ruiz, I. (2003). Evaluation of the psychometric characteristics of the Spanish version of the Hospital Anxiety and Depression Scale. Acta Psychiatrica Scandinavica, 107(3), 216-221.

Renau, V., Gil, F., Oberst, U. \& Carbonell, X. (2015). Internet and Mobile Phone Addiction. In Z. Yan (Ed.), Encyclopedia of Mobile Phone Behavior (pp. 807-817). Hershey, PA: Information Science Reference. doi:10.4018/978-1-4666-8239-9.ch066

Salehan, M., \& Negahban, A. (2013). Social networking on smartphones: When mobile phones become addictive. Computers in Human Behavior, 29(6), 26322639.
Sanchez-Carbonell, X., Beranuy, M., Castellana, M., Chamarro, A. \& Oberst, U. (2008). La adicción a Internet y al móvil: ¿moda o trastorno? Adicciones, 20, 149-159.

Singer, S., Kuhnt, S., Götze, H., Hauss, J., Hinz, A., Liebmann, A., ... Schwarz, R. (2009). Hospital anxiety and depression scale cutoff scores for cancer patients in acute care. British Journal of Cancer, 100(6), 908-912. http://doi.org/10.1038/sj.bjc.6604952

Thomée, S., Härenstam, A., \& Hagberg, M. (2011). Mobile phone use and stress, sleep disturbances, and symptoms of depression among young adults-a prospective cohort study. BMC public health, 11(1), 66.

Young, K. (1998). Internet Addiction: The emergence of a new clinical disorder. Cyberpsychology \& Behavior, $1,237-244$.

Young, K. (2015). The Evolution of Internet Addiction Disorder. En Internet Addiction (pp. 3-17). Switzerland: Springer International Publishing.

\section{Noves tecnologies - ¿Noves patologies? El smartphone i el fear of missing out}

Resum. En el context de les investigacions sobre l'ús intensiu $i$ / o maladaptatiu del telèfon mòbil $i$ de les xarxes socials en línia s'han anat descrivint nous fenòmens que poden ser associats a un malestar psicològic important. El fear of missing out o FoMO (por a perdre's alguna cosa) és un nou constructe psicològic que s'ha descrit com la sensació de malestar quan se sap que altres persones estan realitzant activitats agradables $i$ que un no n'és part. El present estudi investiga la relació entre el grau de FoMO experimentat, els indicadors d'ús problemàtic del telèfon mòbil $i$ els indicadors de malestar psicològic. Mitjançant una anàlisi de regressió lineal jeràrquica es va trobar que tant la conducta addictiva entorn del telèfon mòbil com el FoMO són importants predictors de símptomes psicopatològics, especialment d'ansietat. El fear of missing out es perfila com un correlat important de l'ús desadaptatiu del smartphone $i$ de les xarxes socials en línia.

Paraules clau: xarxes socials en línia; fear of missing out; FoMO, smartphone; telèfon intel-ligent; addicció; ús intensiu

\section{Anexo: Escala de fear of missing out (FoMO-E)}

«A continuación, encontrarás unas afirmaciones sobre tu experiencia del día a día. Usando la escala de 1 a 5, por favor indica en qué medida se ajustan estas afirmaciones a ti, dada tu experiencia en general. Por favor responde aquello que realmente refleje tu experiencia y no lo que piensas como tu experiencia debería ser. Por favor, considera cada pregunta sin tener en cuenta el resto»

1 - nada, 2 - un poco, 3 - moderadamente, 4 - bastante, 5 - mucho

1. A veces me pregunto si dedico demasiado tiempo a estar pendiente de lo que está pasando.

2. Cuando me pierdo una reunión entre amigos, me molesta.

3. Cuando voy de vacaciones, sigo pendiente de lo que mis amigos están haciendo. 
4. Me preocupo cuando me entero de que mis amigos se lo están pasando bien sin mí.

5. Me pongo nervioso cuando no sé qué están haciendo mis amigos.

6. Me da miedo que mis amigos tengan experiencias más gratificantes que yo.

7. Es importante para mí que entienda las bromas que se hacen entre mis amigos.
8. Me molesta cuando pierdo una oportunidad de quedar con amigos.

9. Me da miedo que otras personas tengan experiencias más gratificantes que yo.

10. Cuando me lo paso bien, es importante para mí compartir los detalles en línea (p.ej. actualizando el estatus). 
